\title{
Treatment of End-Stage Osteoarthritis and Concurrent Bony Metastasis to the Distal Femoral Metaphysis with a Modified Total Knee Arthroplasty: A Case Report \& Review of the Literature
}

\author{
Griffin Rechter ${ }^{*}$ and Stephen Brotherton, MD
}

TCU \& UNTHSC School of Medicine, USA

\begin{abstract}
We present a patient with end-stage degenerative osteoarthritis and concurrent osseous metastasis to the distal femoral metaphysis successfully treated with a modified total knee arthroplasty. Improved survivorship in cancer patients with osseous metastasis presents a growing need to explore novel surgical approaches for patients with concomitant endstage osteoarthritis. Currently, endoprosthetic replacement is the intervention most commonly used in the setting of distal femoral metastases. However, total knee arthroplasty is less invasive, economically more conservative, and allows for immediate weight bearing with a lesser risk of prosthetic failure over time. The modified total knee arthroplasty in our patient provided multifactorial benefits: pain relief, tumor resection, immediate weight-bearing, improvement in the overall quality of life, and protection against future pathologic fractures. To our knowledge, this is the first report detailing the use of a modified total knee arthroplasty for end-stage osteoarthritis in the presence of distal femoral metastasis in orthopedic literature.
\end{abstract}

\section{Keywords}

Modified knee arthroplasty, Osteoarthritis, Femur, Metastasis

\section{Introduction}

Metastatic disease is a well-known cause of a rapid deterioration in the quality of life in cancer patients [1]. Similarly, osteoarthritis is the most common pathology affecting the knee joint and is associated with intractable pain and impairments in a patient's quality of life [2]. Patients with metastatic cancer are benefitting from advances in oncology, associated with longer life expectancies. Paralleling the rise in cancer survivorship is the need for total joint arthroplasty [3]. Some studies have estimated a $673 \%$ increase in the demand for TKA by the year 2030 [4]. Thus, there is a mounting need to explore novel approaches to patients requiring TKA in the setting of metastatic disease to the distal femur. Importantly, patients with advanced cancer are considered a high-risk population for arthroplasty. Tumor resection and endoprosthetic replacement are regarded as the preferred method of intervention for metastases to the distal femur [5]. This report details how an alternative intervention, a modified TKA, can improve patients' quality of life when they have end stage osteoarthritis and comorbid metastatic disease to the distal femur. To our knowledge, this is the first report high lighting the utilization of a modified TKA in the treatment of end-stage osteoarthritis, with simultaneous resection of bony metastases from renal cell carcinoma (RCC), and prophylactic pathologic fracture management.

\section{Case History}

An 84-year-old female under went a modified left total knee arthroplasty with a cemented femoral stem to treat her end-stage osteoarthritis in September 2020. She was initially diagnosed with renal cell carcinoma and under went nephrectomy in 2016 with management on nivolumab.

*Corresponding author: Griffin Rechter, TCU \& UNTHSC School of Medicine, 3500 Camp Bowie Boulevard Fort Worth, TX 76107, USA, Tel: +1-713-492-7214

Accepted: February 02, 2021

Published online: February 04, 2021

Citation: Rechter G, Brotherton S (2021) Treatment of EndStage Osteoarthritis and Concurrent Bony Metastasis to the Distal Femoral Metaphysis with a Modified Total Knee Arthroplasty: A Case Report \& Review of the Literature. J Orthop Surg Tech 4(1):239-242

Copyright: (C) 2021 Rechter G, et al. This is an open-access article distributed under the terms of the Creative Commons Attribution License, which permits unrestricted use, distribution, and reproduction in any medium, provided the original author and source are credited. 


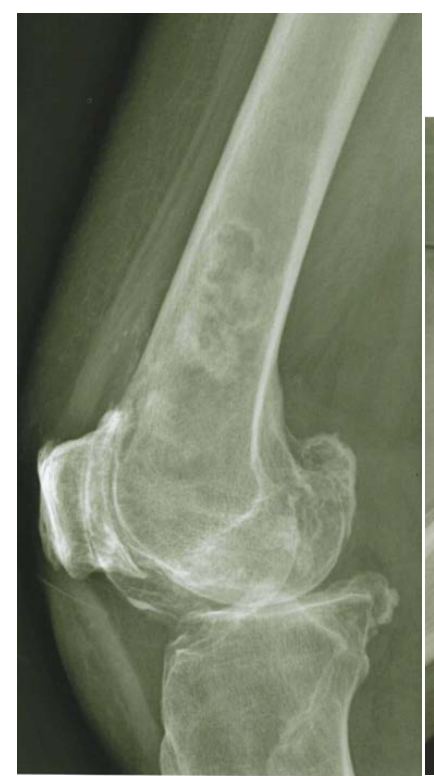

A

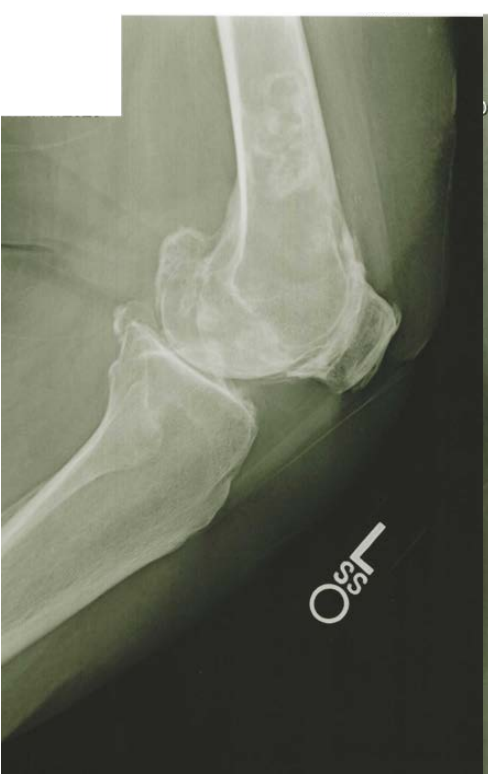

B

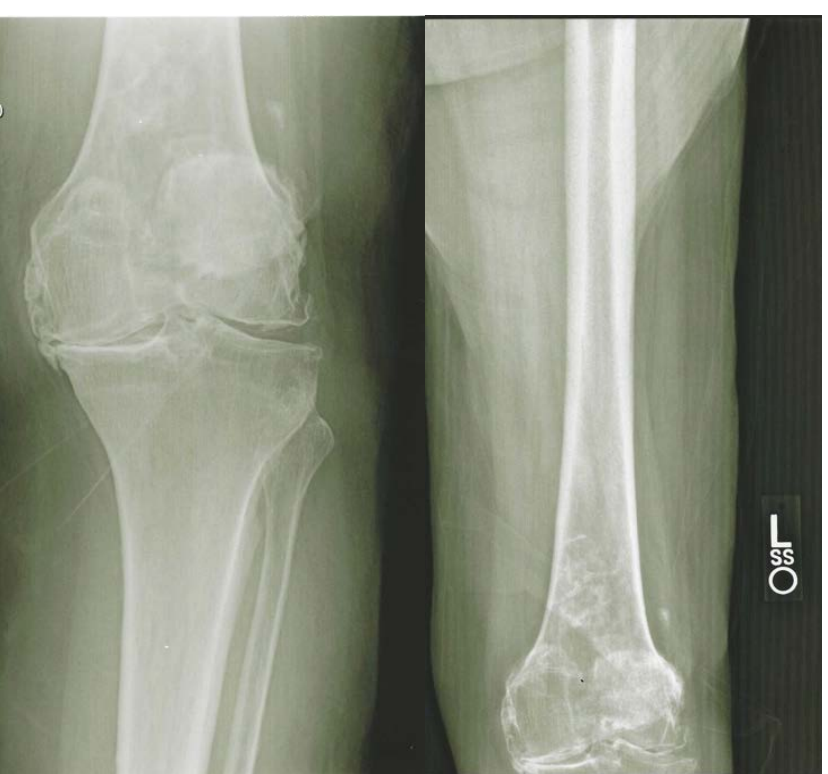

C

Figure 1(A, B, C and D): Pre-Operative Radiographs of AP and lateral of left knee (1A, 1B, 1C) and femur (1D) in 2019.

Originally, the patient presented to our clinic in 2009 with left knee pain and was diagnosed with moderate osteoarthritis. She underwent arthroscopic surgery and managed well for seven years. In 2019 her pain worsened, and radiographs revealed $7^{\circ}$ of varus with moderate degenerative changes in the medial compartment with marked patellofemoral changes. However, the patient was ambulating well and was not a candidate for arthroplasty at that time. She was treated with viscosupplementation injections and achieved $70 \%$ pain relief with improved range of motion.

One year later, she returned with significant left knee pain and impairment in ambulation. Examination showed $15^{\circ}$ of flexion contracture and further medial joint space narrowing. X-rays showed $4^{\circ}$ of varus on standing $A / P$ X-rays with a total deformity of $10^{\circ}$ (Figures $1 \mathrm{a}$, Figures $1 \mathrm{~b}$, Figures $1 \mathrm{c}$ and Figures $1 \mathrm{~d}$ ). She was bone on bone medially, with moderate spurring and substantial patellofemoral disease, making her a reasonable candidate for a TKA. A new osteoblastic lesion in the distal femur was identified on the radiographs and was initially believed to be an enchondroma. After comparing the lesion to prior radiographs from 2019, it was evident that the characteristics of the lesion had changed. The patient had never been previously diagnosed with metastatic cancer. An MRI was ordered and revealed multiple osseous metastases believed to be due to RCC. No biopsy was performed to confirm the origin. The MRI report revealed a $4.2 \times 3.2 \times 16.0 \mathrm{~cm}$ metastatic bony lesion occupying most of the distal half of the left formal shaft extending into the distal metaphysis with no pathologic fracture identified. The MRI also noted severe tricompartmental osteoarthrosis with associated advanced chondromalacia, moderate amount of joint effusion, and diffuse synovitis in the left knee.

\section{Surgical Intervention}

The patient underwent a modified total knee arthroplasty of the left knee in early 2020. Prior to surgery, a thorough discussion amongst her interdisciplinary careteam was conducted to confirm that her disease process and overall health was sufficiently stable to undergo surgical intervention. Careful pre-operative planning was conducted to determine the femoral stem length of $175 \mathrm{~mm}$. A long stem femoral component was used with the Triathlon ${ }^{\circ}$ Total Knee System (Stryker, Kalamazoo, MI, US) [6]. There was no need for a custom prosthesis. The goal was for the stem to extend beyond the metastatic lesion to allow for complete tumor resection, and then to stabilize and fill any bony defects with polymethyl methacrylate (PMMA).

The femoral cuts were performed as in a revision TKA and tibial side cuts were performed as in a primary TKA. The femoral canal was reamed to remove the medullary lesion and ensure endosteal fit. A $175 \mathrm{~mm}$ femoral stem was used, which bypassed the lesion by 5 cortical widths. Cement was then used to fill any skeletal defect from tumor destruction providing stabilization and prevention of further tumor growth. The patient was discharged one day following surgery. Immediate mobilization was possible, and the patient's post-operative course has been unremarkable (Figure $2 a$, Figure $2 b$ and Figure $2 \mathrm{c}$ ).

\section{Discussion}

To our knowledge, this is the first case in orthopedic literature that discusses the utilization of a modified TKA to treat a patient's endstage osteoarthritis and allow for simultaneous resection of bony metastasis. The use of a stemmed femoral component in TKA procedures is primarily used in the revision setting to allow for proper orientation of prosthesis, provide needed stability of the implant, and achieve normal knee kinematics [7]. In this case, the femoral stem component was utilized because the tumor invaded the femoral ca- 
Citation: Rechter G, Brotherton S (2021) Treatment of End-Stage Osteoarthritis and Concurrent Bony Metastasis to the Distal Femoral Metaphysis with a Modified Total Knee Arthroplasty: A Case Report \& Review of the Literature. J Orthop Surg Tech 4(1):239-242

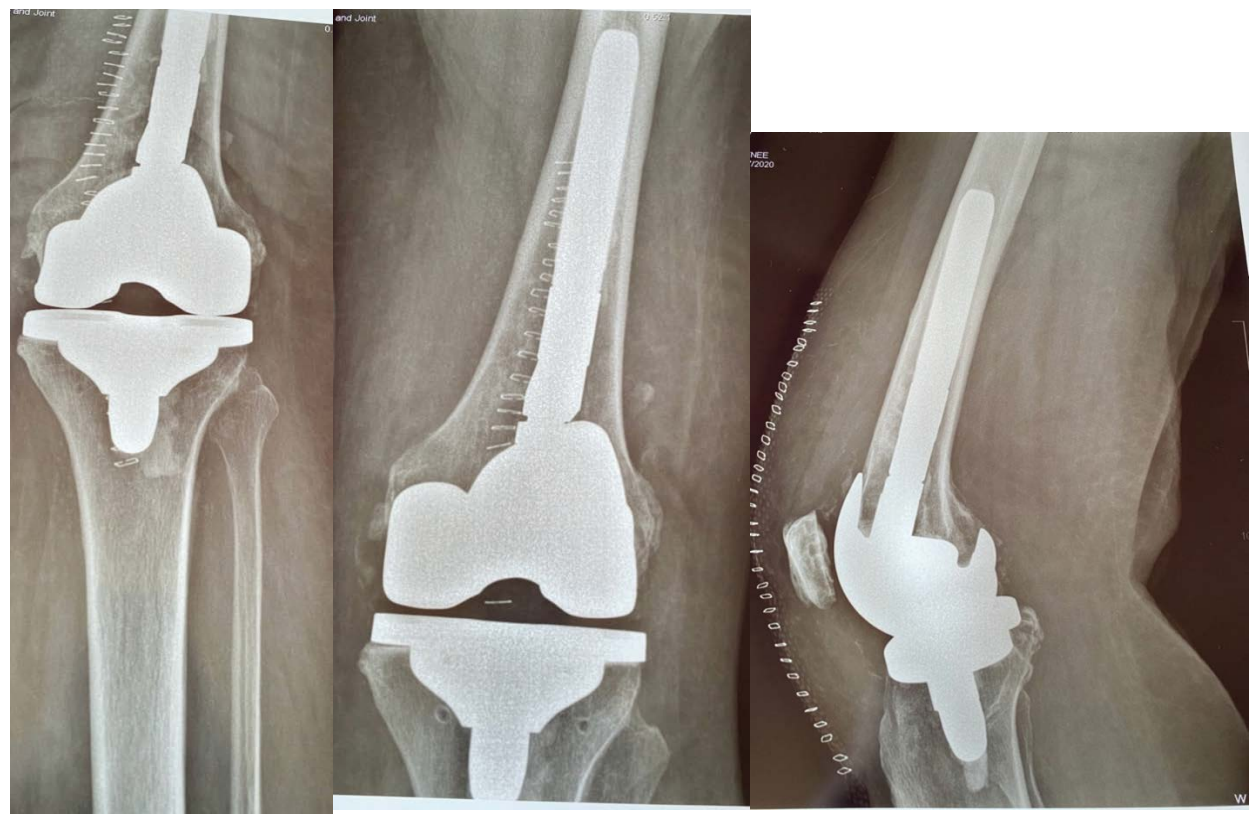

A

B

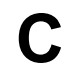

Figure 2(A, B and C): Post-Operative Radiographs: AP and Lateral of $L$ knee in 2020.

nal. Furthermore, the body's innate ability to heal is impaired in pathologic bone. The utilization of a cemented femoral stem allowed for immediate mechanical stability, and a decreased risk for aseptic loosening [8]. Long stem prosthesis also protects the femoral canal from further developing osseous metastasis in the newly revised anatomical location. The tumor's location in the metaphysis was favorable, as large metaphyseal defects are fillable with PMMA [9]. Using a modified TKA contrasts the most commonly used orthopedic intervention with bony metastasis to the distal femur, limb salvage procedures with massive endoprosthesis [10]. However, this patient's presentation allowed for a more conservative approach, a modified TKA, with dual benefit: tumor resection and resolution of end-stage osteoarthritis.

This case also highlights an orthopedic surgeon's role in the setting of skeletal metastasis and concurrent osteoarthritis. Due to improvements in oncology, the survivorship of certain patient subsets with metastatic disease has seen significant improvement. With extended life expectancies, joint pathologies begin to arise at the expense of cell senescence, such as osteoarthritis. An increase in incidence of osteoarthritis in cancer patients with improved life expectancies is expected given that advanced age and a history of cancer are both risk factors for osteoarthritis development [11]. Sloan, et al. stated that "the challenge that confronts patients and surgeons in these difficult situations is whether the complication risk profile after arthroplasty is acceptable and whether sufficient benefit can be derived from the procedure without imparting unnecessary additional suffering" [12]. We believe that patients with metastases to the distal femur with comorbid end-stage osteoarthritis should be considered candidates for a modified total knee arthroplasty as it can offer imme- diate weight-bearing, tumor resection, pathologic fracture prevention, and is fiscally favorable to the utilization of an endoprosthesis $[9,13]$. While massive endoprosthetic reconstruction also provides similar results to the aforementioned benefits of TKA, it is associated with a larger incision, more blood loss, higher risk of neurovascular injury and infection, and carries significant financial burden on patients and the health care system [14-16]. Endoprosthetic reconstruction is also associated with higher rates of failure each subsequent year following surgery [17]. When contemplating when to surgically intervene in cancer patients, overestimating survivorship is more favorable than underestimating it and subsequently requiring a revision surgery in the setting of metastatic disease [18]. In instances where chemotherapy is not indicated, and the cancer is slowly progressive, TKA offers a definitive intervention that will, almost always, last longer than a patient's life expectancy [19].

Lastly, this report also addresses the importance of conducting a thorough patient history and the usefulness of reviewing previous radiographs if available. No prominent findings suggestive of metastatic disease were noted on the initial radiographs taken in 2019. In retrospect, a very subtle blastic lesion is visible in the distal femur. Given that RCC usually presents as a lytic lesion, the diagnosis of an enchondroma was initially suspected. When radiographs were reviewed again in 2020, it was apparent that there were changes in the suspected enchondroma's appearance, which prompted further imaging and identified the lesion to be a metastasis. The tumor's blastic appearance on the radiographs was likely due to the patient's normal regenerative response to osteolysis from the metastatic lesion, which was possible because of the tumor's slow growth. Since metastases can develop after 
Citation: Rechter G, Brotherton S (2021) Treatment of End-Stage Osteoarthritis and Concurrent Bony Metastasis to the Distal Femoral Metaphysis with a Modified Total Knee Arthroplasty: A Case Report \& Review of the Literature. J Orthop Surg Tech 4(1):239-242

a prolonged latency period, especially in slowly progressive cancer subtypes like RCC, osseous lesions can be overlooked and misdiagnosed $[20,21]$. It is difficult to ascertain whether the source of this patient's pain was primarily from end-stage osteoarthritis or the tumor, and it is likely that both played a role in the development of pain. Regardless, this case highlights the necessity to consider a wide differential diagnosis for knee pain with careful attention to a patient's past medical history and imaging.

\section{Conclusion}

In this report we presented a unique case of RCC with osteoblastic metastasis to the distal femur treated with a modified TKA. As the survivorship of patients with cancer improves, unique approaches to the treatment of comorbid conditions are needed. As oncological improvements in the treatment of slow growing cancers, such as RCC, continue to progress there is a need to pay closer attention and conduct a more thorough investigation of bony tumors presenting with unusual radiographic characteristics. Given longer life expectancies in cancer patients and the increasing demand for TKA, the role of the orthopedic surgeon in this patient population is likely to continue to grow and demand extra attention to identify signs of metastasis.

\section{References}

1. Bickels J, Dadia S, Lidar Z (2009) Surgical management of metastatic bone disease. J Bone Joint Surg Am 91: 1503-1516.

2. Anderson AS, Loeser RF (2010) Why is osteoarthritis an age-related disease? Best Pract Res Clin Rheumatol 24: 15-26.

3. Karam JA, Huang RC, Abraham JA, et al. (2015) Total joint arthroplasty in cancer patients. J Arthroplasty 30: 758-761.

4. Kurtz S, Ong K, Lau E, et al. (2007) Projections of primary and revision hip and knee arthroplasty in the United States from 2005 to 2030. J Bone Joint Surg Am 89: 780-785.

5. Haijie L, Dasen L, Tao J, et al. (2018) Implant survival and complication profiles of endoprostheses for treating tumor around the knee in adults: A systematic review of the litera- ture over the past 30 years. J Arthroplasty 33: 1275-1287.e3.

6. Primary Knee Systems. Stryker.

7. Kang SG, Park CH, Song SJ (2018) Stem Fixation in Revision Total Knee Arthroplasty: Indications, Stem Dimensions, and Fixation Methods. Knee Surg Relat Res 30: 187-192.
8. Patel AR, Barlow B, Ranawat AS (2015) Stem length in revision total knee arthroplasty. Curr Rev Musculoskelet Med 8: 407-412.

9. Johnson JD, Wyles CC, Perry KI, et al. (2020) Outcomes of knee arthroplasty for primary treatment of pathologic peri-articular fractures of the distal femur and proximal tibia. Int Orthop 44: 187-193.

10. Soeharno H, Povegliano L, Choong PF (2018) Multimodal treatment of bone metastasis-A surgical perspective. Front Endocrinol (Lausanne) 9: 518.

11. Heidari B (2011) Knee osteoarthritis prevalence, risk factors, pathogenesis and features: Part I. Caspian J Intern Med 2: 205212.

12. Sloan M, Lee GC (2020) Mortality and complications in patients with metastatic disease after primary total hip and total knee arthroplasty. J Arthroplasty 35: 3512-3516.

13. Camnasio F, Scotti C, Peretti GM, et al. (2008) Prosthetic joint replacement for long bone metastases: Analysis of 154 cases. Arch Orthop Trauma Surg 128: 787-793.

14. Wilson RJ, Sulieman LM, VanHouten JP, et al. (2017) Cost-utility of osteoarticular allograft versus endoprosthetic reconstruction for primary bone sarcoma of the knee: A markov analysis. J Surg Oncol 115: 257-265.

15. Henderson ER, Groundland JS, Pala E, et al. (2011) Failure mode classification for tumor endoprostheses: Retrospective review of five institutions and a literature review. J Bone Jt Surg Ser Am 93: 418-429.

16. Lotke PA, Faralli VJ, Orenstein EM, et al. (1991) Blood loss after total knee replacement. Effects of tourniquet release and continuous passive motion. J Bone Jt Surg Am 73: 1037-1040.

17. Yang R-S (2004) Endoprosthesis-related complications after limb-salvage operation of malignant bone tumors around the knee. Biomed Eng Appl Basis Commun 16: 133-142.

18. Agarwal MG, Nayak P (2015) Management of skeletal metastases: An orthopaedic surgeon's guide. Indian J Orthop 49: 83-100.

19. Smith EM, Kursh ED, Makley J, et al. (1992) Treatment of osseous metastases secondary to renal cell carcinoma. J Urol 148: 784-787.

20. Swanson DA, Orovan WL, Johnson DE, et al. (1981) Osseous metastases secondary to renal cell carcinoma. Urology 18: 556-561.

21. Gould CF, Ly JQ, Lattin GE, et al. (2007) Bone tumor mimics: Avoiding misdiagnosis. Curr Probl Diagn Radiol 36: 124-141.

DOI: $10.36959 / 453 / 554$

Copyright: (C) 2021 Rechter G, et al. This is an open-access article distributed under the terms of the Creative Commons Attribution License, which permits unrestricted use, distribution, and reproduction in any medium, provided the original author and source are credited. 